\title{
Macular sensitivity and morphology after intravitreal injection of triamcinolone acetonide for macular edema secondary to central retinal vein occlusion
}

\author{
This article was published in the following Dove Press journal: \\ Clinical Ophthalmology \\ 16 October 2012 \\ Number of times this article has been viewed
}

\author{
Hidetaka Noma' \\ Tatsuya Mimura ${ }^{2}$ \\ 'Department of Ophthalmology, \\ Yachiyo Medical Center, Tokyo \\ Women's Medical University, Chiba, \\ 2Department of Ophthalmology, \\ Medical Center East, Tokyo Women's \\ Medical University, Tokyo, Japan
}

\begin{abstract}
It has been reported that microperimetry is effective for evaluating macular function. The aim of this paper is to report and discuss changes seen in macular sensitivity and macular thickness after intravitreal injection of triamcinolone acetonide (IVTA) in two patients with macular edema and central retinal vein occlusion (CRVO). Two eyes from two patients with CRVO (one each with ischemic and nonischemic CRVO) received IVTA for macular edema. Microperimetry was performed to measure macular sensitivity within the central 4, 10, and 20 degree fields, while the macular thickness within these fields was measured by optical coherence tomography. The mean macular thickness and macular sensitivity within the central 4, 10, and 20 degree fields improved after IVTA in the patient with nonischemic CRVO. In contrast, the mean macular thickness and sensitivity within the central 4, 10, and 20 degree fields showed little change after IVTA in the patient with ischemic CRVO. IVTA improves macular sensitivity and morphology in nonischemic CRVO, so further evaluation of this treatment is justified.
\end{abstract}

Keywords: central retinal vein occlusion, macular edema, triamcinolone acetonide, macular sensitivity

\section{Introduction}

Macular edema is the most serious complication of central retinal vein occlusion (CRVO) with respect to the visual prognosis. We have previously reported that various inflammatory factors may have a role in the pathogenesis of macular edema associated with CRVO, suggesting that inflammation may contribute to the onset and/or exacerbation of macular edema in these patients. ${ }^{1,2}$ The Standard Care Versus Corticosteroid for Retinal Vein Occlusion study ${ }^{3}$ demonstrated that intravitreal injection of triamcinolone acetonide (IVTA) maintains improvement in visual acuity and macular edema for 12 months in patients with CRVO. However, that study only measured visual acuity (which purely reflects foveal function) to evaluate the visual prognosis, even though macular edema usually involves the larger macular area. The Micro Perimeter 1 (MP-1) is an instrument that can evaluate both the fovea and the larger macular area by combining digital fundus imaging with automated perimetry. ${ }^{4}$ We recently demonstrated that retinal thickness and retinal volume are correlated with both retinal sensitivity and best-corrected visual acuity (BCVA) in patients with CRVO and macular edema. ${ }^{5}$ Here, we report on the changes in macular sensitivity and macular thickness after IVTA was done to treat macular edema in two patients with CRVO.
Correspondence: Hidetaka Noma Department of Ophthalmology, Yachiyo Medical Center, Tokyo Women's Medica University, 477-96, Owada-shinden, Yachiyo, Chiba 276-8524, Japan

Tel +8I 474506000

Fax +8I 474587047

Email noma-hide@umin.ac.jp 


\section{Materials and methods}

The treatment described herein was reviewed by our institutional ethics committee and adhered to the tenets of the Declaration of Helsinki. Written informed consent was obtained from both patients, who had CRVO and macular edema, and underwent IVTA for one eye each. In addition, consent to publishing the study results was obtained from both patients.

Both patients received IVTA under local anesthesia. For intravitreal therapy, $4.0 \mathrm{mg}$ of triamcinolone acetonide was administered in a volume of $0.1 \mathrm{~mL}$ via the pars plana at 3-4 mm posterior to the limbus. The injection was performed with a sterile technique, and prophylactic topical antibiotics were applied for one week afterwards. Both patients were followed up for at least 6 months postoperatively. Macular sensitivity was investigated by microperimetry (MP-1 microperimeter; Nidek, Gamagori, Japan), and macular thickness was measured by optical coherence tomography (Zeiss Stratus OCT3, Carl Zeiss Meditec, Dublin, CA) before and at 3 and 6 months after IVTA.

As previously reported, ${ }^{5}$ a retinal thickness map and a retinal volume map were obtained by scanning $6 \times 6 \mathrm{~mm}$ $(20 \times 20$ degrees $)$ areas of the macular region, which was divided into nine subfields, ie, the fovea, superior inner macula, nasal inner macula, inferior inner macula, temporal inner macula, superior outer macula, nasal outer macula, inferior outer macula, and temporal outer macula. The mean macular thickness at the one subfield (fovea) covering the central $1 \times 1 \mathrm{~mm}(4 \times 4$ degrees), at five subfields (fovea, superior inner, nasal inner, inferior inner, and temporal inner) covering the central $3 \times 3 \mathrm{~mm}(10 \times 10$ degrees $)$, and at all nine subfields covering the entire central $6 \times 6 \mathrm{~mm}$ $(20 \times 20$ degrees $)$ were thus determined.

As previously reported, ${ }^{5}$ mean retinal sensitivity was calculated from the sensitivity of each of the nine subfields on the retinal map generated by optical coherence tomography. In addition, mean macular sensitivity was measured at five locations covering the central 4 degree field, 29 locations covering the central 10 degree field (five subfields, ie, fovea, superior inner, nasal inner, inferior inner, and temporal inner), and 57 locations covering the entire central 20 degree field (all nine subfields).

\section{Case report I}

A 72-year-old man presented to our hospital complaining of loss of vision in his right eye from one month earlier. He was otherwise in excellent health and had no history of illness. BCVA was 20/100 for his right eye and 20/20 for his left eye, and intraocular pressure was $11 \mathrm{mmHg}$ in both eyes. An afferent pupillary defect was detected in his right eye. Anterior segment examination did not demonstrate any neovascularization or other changes. In the left eye, the fundus was normal. However, the fundus of the right eye showed flame-shaped intraretinal hemorrhages in all four quadrants. Findings on fluorescein angiography (Figure 1) and electroretinography (a slight decrease of the b wave amplitude) were compatible with nonischemic CRVO.

IVTA was performed 2 months after the onset of symptoms. The mean macular thickness within the 10 degree and 20 degree fields showed a decrease at 3 and 6 months after IVTA (Figure 2). Visual acuity was also improved after 3 and 6 months (Figure 3). Although the mean macular sensitivity within the central 4, 10, and 20 degree fields was increased at 3 months after IVTA, it showed a decrease at 6 months compared with 3 months (Figures 4 and 5). Intraocular pressure remained normal in both eyes and neovascular glaucoma was not detected. There was also no progression of cataract after IVTA, and infectious endophthalmitis did not occur.

\section{Case report 2}

A 70-year-old woman presented to our hospital with loss of vision in her right eye for 2 months. Her health was excellent and she had had prior illnesses. BCVA was 20/200 for her right eye and 20/20 for her left eye. An afferent pupillary defect was found in her right eye, the intraocular pressure was $10 \mathrm{mmHg}$, and there was no anterior segment neovascularization. Examination of the right fundus revealed flame-shaped intraretinal hemorrhages in all four quadrants, while the fluorescein angiography (Figure 1) and electroretinographic findings (a marked decrease of the $b$ wave amplitude) were compatible with a diagnosis of ischemic CRVO.

Four months after the onset of symptoms, IVTA was performed. The mean macular thickness within the central 4, 10 , and 20 degree fields was reduced at 3 months after IVTA,

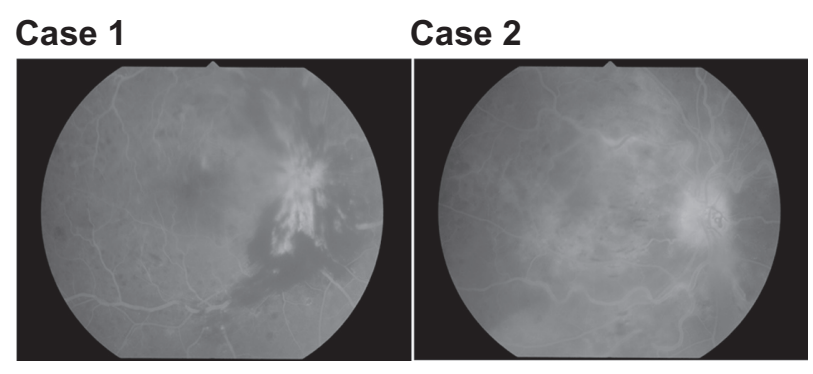

Figure I Fluorescein angiography. Case I: macular edema without macular ischemia. Case 2: macular edema associated with ischemia of the macular region. 
but was increased again at 6 months compared with 3 months (Figure 2). Visual acuity did not improve after either 3 or 6 months (Figure 3). Moreover, mean macular sensitivity within the central 4, 10, and 20 degree fields showed little change after 3 or 6 months (Figures 4 and 5). Intraocular pressure remained normal in both eyes and neovascular glaucoma was not detected after IVTA. There was also no progression of cataract and no infectious endophthalmitis.

\section{Discussion}

Case 1 was followed up for one month and then referred to us. We found decreased visual acuity, and treatment was started one month later, ie, in the second month. Case 2 was referred
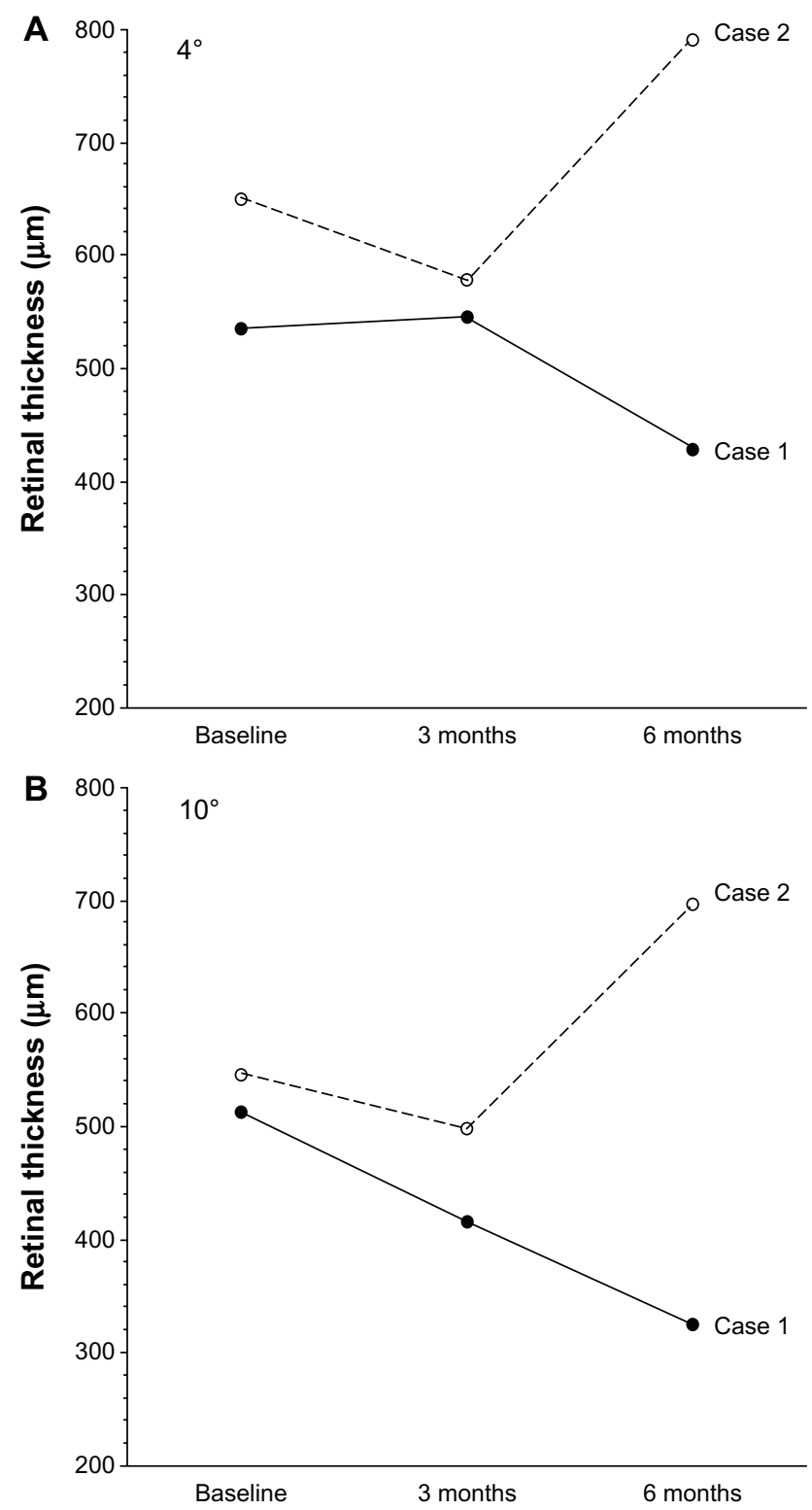

Figure 2 (Continued)

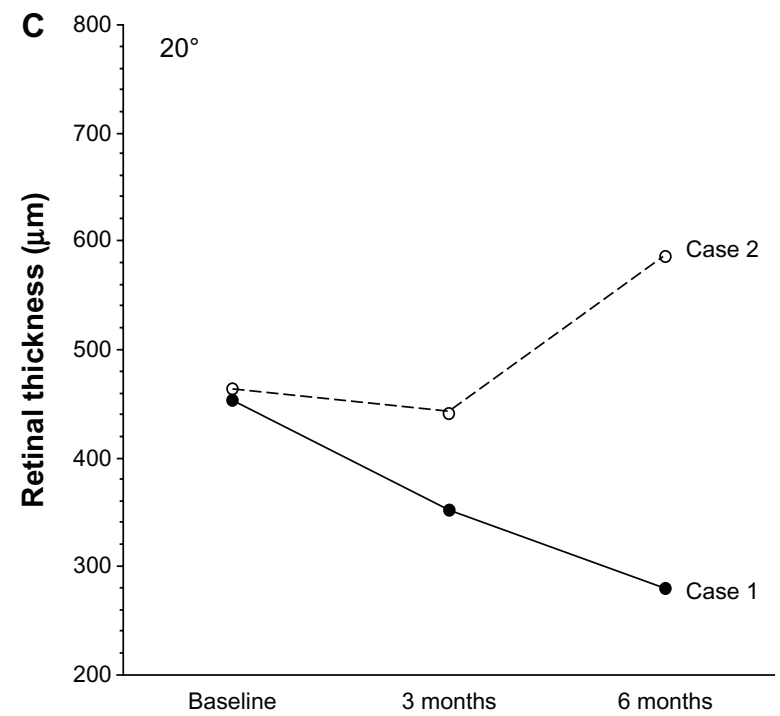

Figure 2 Changes in mean macular thickness (A-C) after intravitreal injection of triamcinolone acetonide in two patients with branch retinal vein occlusion and macular edema. (A) Case I: mean macular thickness within the central 4 degree field is reduced after 3 and 6 months. Case 2: mean macular thickness within the central 4 degree field is reduced after 3 months, but increased at 6 months compared with at 3 months. (B) Case I: mean macular thickness within the 10 degree field is reduced after 3 and 6 months. Case 2: mean macular thickness within the 10 degree field is reduced after 3 months, but increased at 6 months compared with at 3 months. (C) Case I: mean macular thickness within the 20 degree field is reduced after 3 and 6 months. Case 2: mean macular thickness within the 20 degree field is reduced after 3 months, but increased at 6 months compared with at 3 months.

to us 2 months after the onset of symptoms. This patient was also observed for one month without improvement, and IVTA was performed in the fourth month.

In case 1, MP-1 changes were detected before visual acuity decreased and retinal thickness increased. Considering this

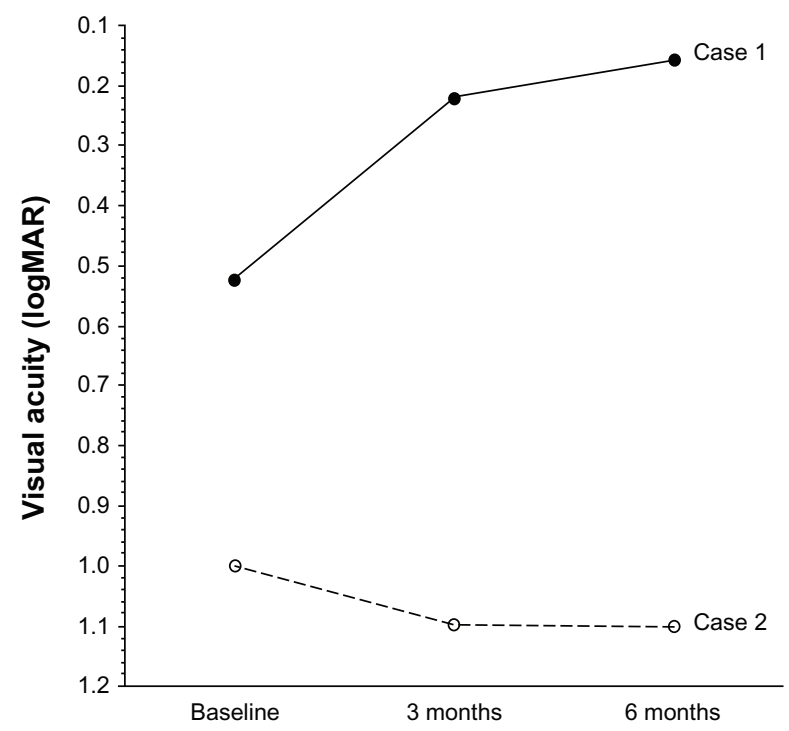

Figure 3 Changes in best-corrected visual acuity after intravitreal injection of triamcinolone acetonide in two patients with branch retinal vein occlusion and macular edema. Case I: best-corrected visual acuity is improved after 3 and 6 months. Case 2: best-corrected visual acuity is not improved after 3 or 6 months. 

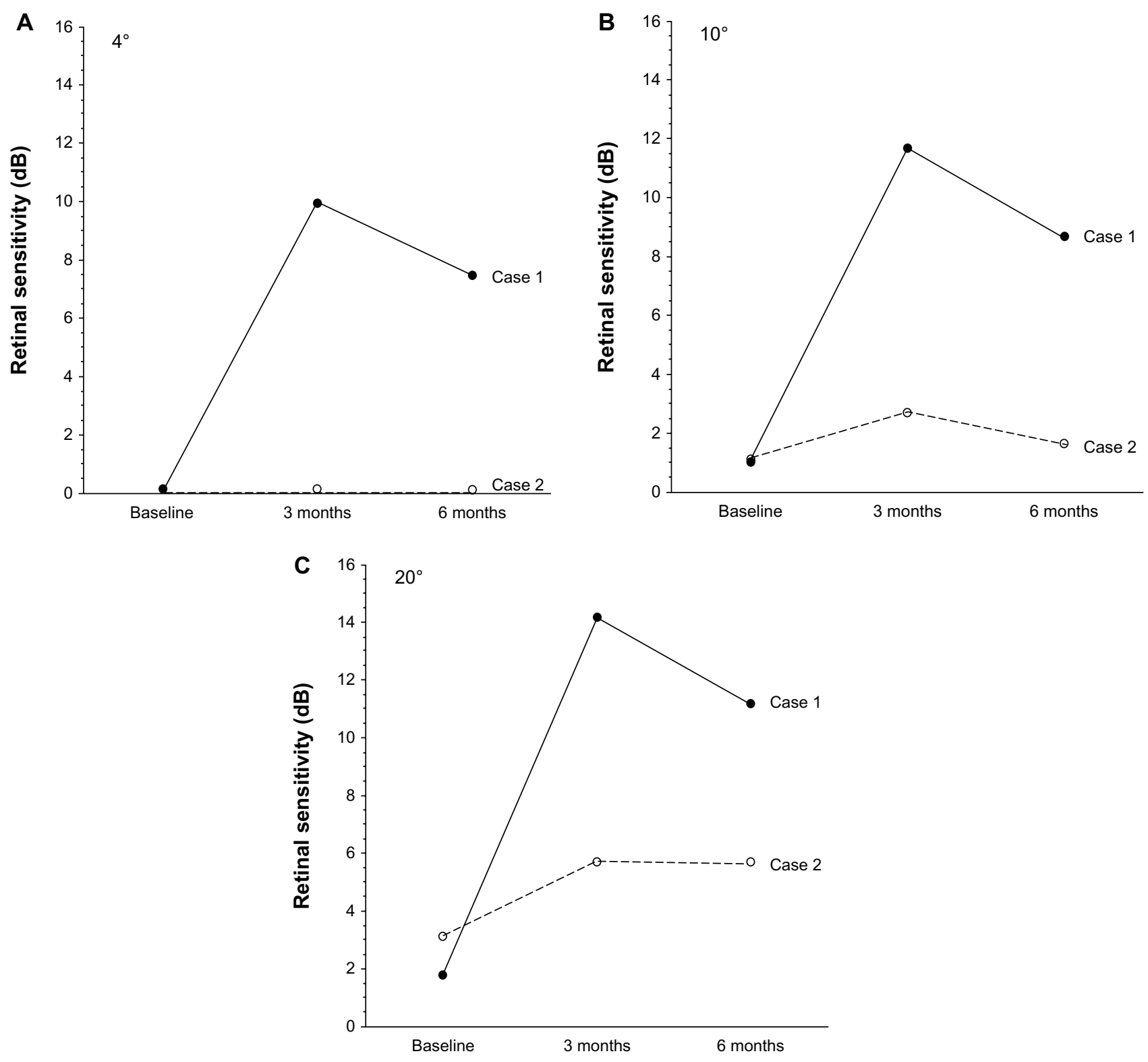

Figure 4 Changes in macular sensitivity $(\mathbf{A}-\mathbf{C})$ after intravitreal injection of triamcinolone acetonide in two patients with branch retinal vein occlusion and macular edema. (A) Case I: mean macular sensitivity within the central 4 degree field is increased after 3 months, but decreased at 6 months compared with at 3 months. Case 2: mean macular sensitivity within the central 4 degree field is not improved after 3 or 6 months. (B) Case I: mean macular sensitivity within the 10 degree field is increased after 3 months, but decreased at 6 months compared with at 3 months. Case 2: mean macular sensitivity within the 10 degree field is almost unchanged after 3 and 6 months. (C) Case I: mean macular sensitivity within the 20 degree field is increased after 3 months, but decreased at 6 months compared with at 3 months. Case 2: mean macular sensitivity within the 20 degree field is almost unchanged after 3 and 6 months.

course, changes in MP-1 may be a prelude to functional or structural deterioration. Such changes may be attributable to fluctuation.

In the first patient, who had nonischemic CRVO, mean macular thickness within the central 4, 10, and 20 degree fields showed a decrease at 3 and 6 months after IVTA. Triamcinolone acetonide may improve macular edema by decreasing retinal capillary permeability via an influence on tight junctions ${ }^{6}$ or it could inhibit the vascular endothelial growth factor (VEGF) signaling cascade that increases microvascular permeability. ${ }^{7,8}$ Corticosteroids may also inhibit the production of inflammatory factors ${ }^{9,10}$ promoting leukocyte adhesion and breakdown of the blood-retinal barrier, thus preventing an increase in vascular permeability. ${ }^{11}$ Therefore, macular thickness may have been reduced by IVTA, resulting in improvement of macular sensitivity. However, the mean macular sensitivity within the central 4, 10, and 20 degree fields was lower at 6 months than at 3 months after IVTA, although macular thickness maintained its improvement at 6 months. This may have been due to differences in the 


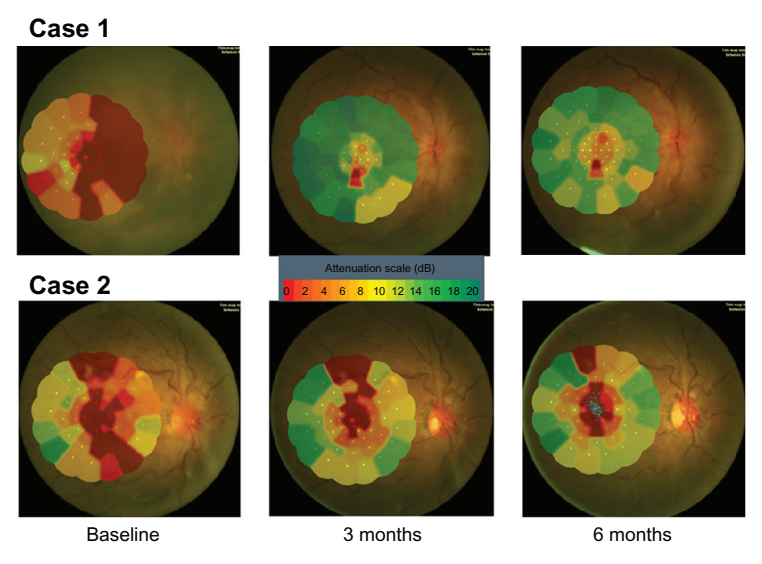

Figure 5 Macular sensitivity map obtained by microperimetry.

Notes: Macular sensitivity maps were obtained using the Micro Perimeter I before intravitreal injection of triamcinolone acetonide, as well as at 3 and 6 months after triamcinolone acetonide. Changes in macular sensitivity are shown in Figure 3.

temporal profile for improvement of macular thickness and macular sensitivity.

In the second patient, mean macular sensitivity within the central 4 degrees was $0 \mathrm{~dB}$. The dynamic range of most microperimeters is rather narrow compared with that of standard automatic perimetry. Therefore, it is considered that the true sensitivity in the second case was 0 due to absolute scotoma. In addition, macular ischemia may decrease macular function, as well as macular edema.

A different outcome was seen in our patient with ischemic CRVO, with both macular thickness and macular sensitivity showing little change at 3 or 6 months after IVTA. We have previously demonstrated that vitreous fluid levels of inflammatory factors are significantly higher in patients with ischemic CRVO than in patients with nonischemic CRVO. ${ }^{1,2}$ Therefore, it is possible that the inflammatory factor levels are too high in patients with ischemic CRVO for one IVTA procedure to have much effect, resulting in minimal change in mean macular thickness and macular sensitivity after 3 and 6 months. However, mean macular sensitivity within the 20 degree field was improved after 3 and 6 months compared with that for the central 4 degree field. This suggests that retinal function may recover more rapidly in the macular region outside the fovea in patients with ischemic CRVO.

Recently, Senturk et al ${ }^{12}$ reported that short-term improvement in retinal sensitivity can be achieved by IVTA in patients with nonischemic CRVO and macular edema. Although their study did not include any patients with ischemic CRVO, this finding is in agreement with the outcome for the patient described in our first case report. It has also been reported that macular function improves after intravitreal injection of an anti-VEGF agent in patients with macular edema secondary to retinal vein occlusion. ${ }^{13,14}$ Accordingly, a larger prospective investigation is required to confirm the changes in macular function after IVTA or anti-VEGF therapy in patients suffering from CRVO with or without ischemia. In conclusion, IVTA achieves improvement in macular sensitivity and morphology in nonischemic CRVO, suggesting that further evaluation of this treatment is justified.

\section{Disclosure}

The authors report no conflicts of interest in this work.

\section{References}

1. Noma H, Funatsu H, Mimura T, Harino S, Hori S. Vitreous levels of interleukin-6 and vascular endothelial growth factor in macular edema with central retinal vein occlusion. Ophthalmology. 2009;116(1):87-93.

2. Noma H, Funatsu H, Harino S, Mimura T, Eguchi S, Hori S. Vitreous inflammatory factors in macular edema with central retinal vein occlusion. Jpn J Ophthalmol. 2011;55(3):248-255.

3. Ip MS, Scott IU, VanVeldhuisen PC, et al. A randomized trial comparing the efficacy and safety of intravitreal triamcinolone with observation to treat vision loss associated with macular edema secondary to central retinal vein occlusion: the Standard Care vs Corticosteroid for Retinal Vein Occlusion (SCORE) study report 5. Arch Ophthalmol. 2009; 127(9):1101-1114.

4. Noma H, Funatsu H, Mimura T, Harino S, Shimada K. Functionalmorphologic correlates in patients with branch retinal vein occlusion and macular edema. Retina. 2011;31(10):2102-2108.

5. Noma H, Mimura T, Shimada K. Retinal function and morphology in central retinal vein occlusion with macular oedema. Curr Eye Res. September 19, 2012. [Epub ahead of print.]

6. Antonetti DA, Wolpert EB, DeMaio L, Harhaj NS, Scaduto RC Jr. Hydrocortisone decreases retinal endothelial cell water and solute flux coincident with increased content and decreased phosphorylation of occludin. J Neurochem. 2002;80(4):667-677.

7. Zhang X, Bao S, Lai D, Rapkins RW, Gillies MC. Intravitreal triamcinolone acetonide inhibits breakdown of the blood-retinal barrier through differential regulation of VEGF-A and its receptors in early diabetic rat retinas. Diabetes. 2008;57(4):1026-1033.

8. McAllister IL, Vijayasekaran S, Chen SD, Yu DY. Effect of triamcinolone acetonide on vascular endothelial growth factor and occludin levels in branch retinal vein occlusion. Am J Ophthalmol. 2009;147(5):838-846.

9. Kurtz RM, Elner VM, Bian ZM, Strieter RM, Kunkel SL, Elner SG. Dexamethasone and cyclosporin A modulation of human retinal pigment epithelial cell monocyte chemotactic protein-1 and interleukin-8. Invest Ophthalmol Vis Sci. 1997;38(2):436-445.

10. Mizuno S, Nishiwaki A, Morita H, Miyake T, Ogura Y. Effects of periocular administration of triamcinolone acetonide on leukocyteendothelium interactions in the ischemic retina. Invest Ophthalmol Vis Sci. 2007;48(6):2831-2836.

11. Miyamoto K, Khosrof S, Bursell SE, et al. Prevention of leukostasis and vascular leakage in streptozotocin-induced diabetic retinopathy via intercellular adhesion molecule-1 inhibition. Proc Natl Acad Sci USA. 1999;96(19):10836-10841.

12. Senturk F, Ozdemir H, Karacorlu M, Karacorlu SA, Uysal O. Microperimetric changes after intravitreal triamcinolone acetonide injection for macular edema due to central retinal vein occlusion. Retina. 2010;30(8):1254-1261.

13. Kriechbaum K, Prager F, Geitzenauer W, et al. Association of retinal sensitivity and morphology during antiangiogenic treatment of retinal vein occlusion over one year. Ophthalmology. 2009;116(12):2415-2421.

14. Yamaike N, Tsujikawa A, Sakamoto A, et al. Retinal sensitivity after intravitreal injection of bevacizumab for the treatment of macular edema secondary to retinal vein occlusion. Retina. 2009;29(6):757-767. 


\section{Publish your work in this journal}

Clinical Ophthalmology is an international, peer-reviewed journal covering all subspecialties within ophthalmology. Key topics include: Optometry; Visual science; Pharmacology and drug therapy in eye diseases; Basic Sciences; Primary and Secondary eye care; Patient Safety and Quality of Care Improvements. This journal is indexed on

Submit your manuscript here: http://www.dovepress.com/clinical-ophthalmology-journal
PubMed Central and CAS, and is the official journal of The Society of Clinical Ophthalmology (SCO). The manuscript management system is completely online and includes a very quick and fair peer-review system, which is all easy to use. Visit http://www.dovepress.com/ testimonials.php to read real quotes from published authors. 